\title{
Reuse of Neutral Red-loaded Wheat Straw for Adsorption of Congo Red from Solution in a Fixed-bed Column
}

\author{
Jinfang Miao ${ }^{a}$, Lifang Liu ${ }^{b}$, Yizhen Lu ${ }^{c}$, Yueqi Song ${ }^{d}$, Runping Han ${ }^{e_{*}}$ \\ School of Chemistry and Molecular Engineering, Zhengzhou University, Kexue Road 100, \\ Zhengzhou, 450001, PR China \\ aemail:974585025@qq.com, bemail: 2206511017@qq.com, 'email: 1363493238@qq.com, ${ }^{\mathrm{d} e m a i l}$ \\ songyaoqi@126.com, ${ }^{e}$ * Corresponding author email: rphan67@zzu.edu.cn
}

\begin{abstract}
Keywords: Neutral red-loaded wheat husk; Column adsorption; Congo Red; Thomas model Abstract. In this study, wheat straw loaded with neutral red was reused to adsorb congo red (CR) from aqueous solution in a fixed-bed column. Compared to wheat straw, adsorption capacity of NR-loaded wheat straw towards CR was significantly higher. The the flow rate, the influent concentration of CR and bed depth significantly affect the shape of breakthrough curves. The Thomas model was applied to fit the experimental data and the results showed that it was available to predict the breakthrough curves and to determine the characteristic parameters of the column useful for process design using nonlinear regression.
\end{abstract}

\section{Introduction}

With the increasing concern on environment protection in recent years, removal of pollutants is gaining attention. Dyes, which is the main component in the effluent of textile, paper, plastic, food and cosmetic industries, is an important contaminants in water bodies [1]. Color is a visible pollutant and the presence of even a very minute amount of coloring substance makes it undesirable due to its appearance. Diverse techniques, including adsorption, flocculation, oxidation and electrolysis, have been employed for removal of dyes from wastewaters. Among these methods, adsorption is superior since it is more efficient and economical for removal of refractory pollutants (including dyes) [2]. The widely used and effective physical method in industry is activated carbon, but the high cost limited its widespread use. Therefore, several biological materials, especially agricultural residues including peanut husk, wheat straw, rice husk, chaff, tree leaf, etc [3-8], have been investigated for removal of dyestuff in our laboratory. There were also several reviews about this field [9].

As a type of renewable crop wastes, wheat straw is not only abundant in nature but rich in reactive groups existing in cellulose, hemicellulose and lignin structures, which are effective as adsorbent [10]. For wheat straw, there is higher adsorption capacity for cationic pollutants and lower adsorption capacity for anionic pollutants. Furthermore, after adsorption of dyes, regeneration and reuse of the used or exhausted adsorbents is a problem. Conventionally, large amount of solvent is applied as eluent to wash and recover adsorbents for recycle use. However, the generated waste eluent usually requires further treatment to avoid the secondary pollution. Recently, a novel way to make use of used adsorbents has been raised in reported literatures [11]. In this work, the final cationic dyes loaded adsorbents were tried to revmoval anionic dye, Conogo red(CR) from solution in fixed-bed column.

\section{Materials and methods}

Materials. The wheat straw (WS) selected in the present investigation was obtained from the local countryside. The collected materials were washed with distilled water several times to remove all the dirt particles. The washed material was dried in an oven at $373 \mathrm{~K}$ for a period of $24 \mathrm{~h}$ and then ground and screened through a set of sieves to get the geometrical sizes (20 to 40) mesh for column studies. The solutions of NR (50 mg/L) were prepared in distilled water ( $\mathrm{pH}$ of NR solution 6.5). The stock solutions of CR $(500 \mathrm{mg} / \mathrm{L})$ were prepared in distilled water. All working solutions were prepared by diluting the stock solution with distilled water. The $\mathrm{pH}$ of CR solution was near 3.0 and 
was not adjusted.

Methods. Fixed-bed column adsorption experiments were carried out using WS packed into a glass column $(1.0 \mathrm{~cm}$ inner diameter and $25 \mathrm{~cm}$ in height). The experiments were conducted in down flow mode through the fixed-bed with a peristaltic pump. The temperatures of all experiments were $293 \mathrm{~K}$. NR-loaded columns were obtained as following: the mass of adsorbent in the column was 5, 8 and $11 \mathrm{~cm}$. The NR solution of $50 \mathrm{mg} / \mathrm{L}$ was pumped to the column at $10 \mathrm{~mL} / \mathrm{min}$. Also, the experiments of three different bed depths, $5 \mathrm{~cm}, 8 \mathrm{~cm}, 11 \mathrm{~cm}$, were operated at the same influent NR concentration $(50 \mathrm{mg} / \mathrm{L})$ and flow rate $(8 \mathrm{~mL} / \mathrm{min})$. After adsorption, the distilled water was pumped through the column till the effluent was not color and NR-loaded WS columns were obtained. Then CR solution was through the column at various conditions.

Samples were collected at regular intervals in all the adsorption. The concentration of CR in effluent was analyzed using a UV/Vis-3000 spectrophotometer by monitoring the absorbance changes at a wavelength of maximum absorbance $(500 \mathrm{~nm})$. The breakthrough curves can be obtained according to $C_{t} / C_{0}$ to time $t$.

The maximum column capacity, $q_{\text {total }}(\mathrm{mg})$, for a given feed concentration and flow rate is equal to the area under the plot of the adsorbed NR concentration $C_{\mathrm{ad}}\left(C_{\mathrm{ad}}=C_{0}-C\right)(\mathrm{mg} / \mathrm{L})$ versus time (min) and is calculated from Eq(1) [12]:

$$
q_{\text {total }}=\frac{v A}{1000}=\frac{v}{1000} \int_{t=0}^{t=t_{\text {total }}} C_{a d} d t
$$

where $t_{\text {total }}, v$ and $A$ are the total flow time $(\mathrm{min})$, volumetric flow rate $(\mathrm{mL} / \mathrm{min})$ and the area under the breakthrough curve, respectively.

The equilibrium uptake $\left(q_{\mathrm{e}}\right)$, the weight of CR adsorbed per unit dry weight of adsorbent (mg/g) in the column, is calculated as following:

$$
q_{\mathrm{e}}=\frac{q_{\text {total }}}{x}
$$

where $x$ is the total dry weight of WS in column (g).

Thomas model is one of the most general and widely used models in column performance theory. The expression by Thomas for an adsorption column is given as follows [12,13]:

$$
\frac{C_{\mathrm{t}}}{C_{0}}=\frac{1}{1+\exp \left(k_{\mathrm{Th}} q_{0} x / v-k_{\mathrm{Th}} C_{0} t\right)}
$$

where $k_{\mathrm{Th}}$ is the Thomas rate constant ( $\mathrm{mL} / \mathrm{min} \mathrm{mg}$ ); $q_{0}$ is the equilibrium CR uptake per $\mathrm{g}$ of the adsorbent (mg/g); $x$ is amount of adsorbent in the column $(\mathrm{g}) ; v$ is flow rate $(\mathrm{mL} / \mathrm{min})$.

\section{Results and discussions}

Effect of influent CR concentration on breakthrough curves. The breakthrough curves at different $\mathrm{CR}$ concentration were shown in Fig. 1. It was illustrated that the breakthrough time decreased with increasing influent CR concentration. At lower influent concentrations, breakthrough occurred slower. As influent concentration increased, sharper breakthrough curves were obtained. These results demonstrate that the change of concentration gradient affects the saturation rate and breakthrough time. 


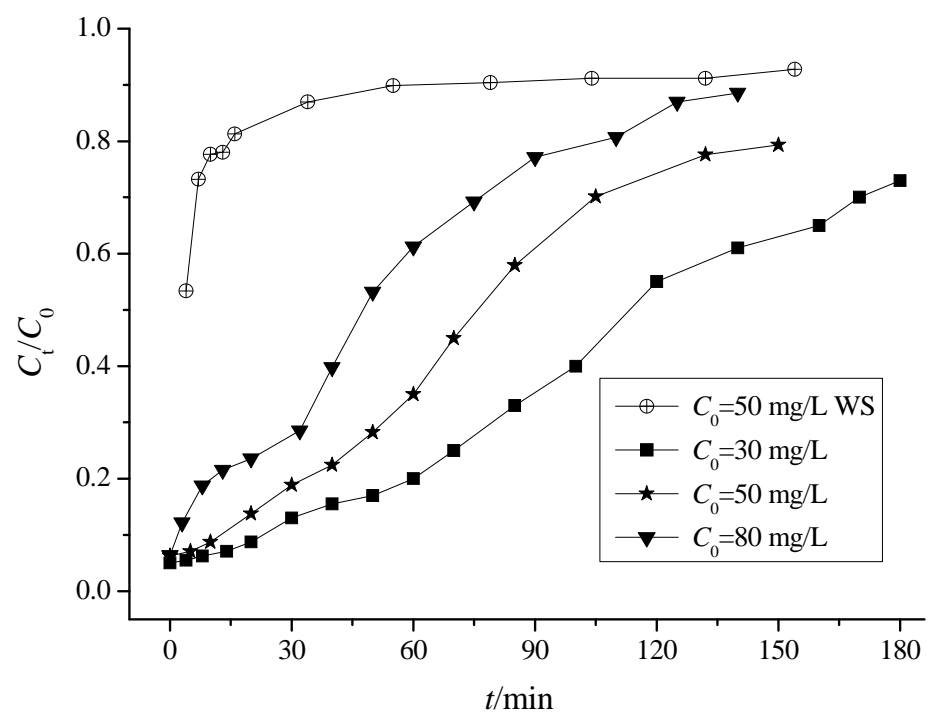

Fig. 1. Effect of CR concentration on breakthrough curves and comparison of breakthrough curves between WS and NR-loaded WS ( $v=10 \mathrm{~mL} / \mathrm{min}, \mathrm{Z}=8 \mathrm{~cm})$.

Fig. 1 also showed the breakthrough curve of WS toward CR adsorption. It was observed that the slope of breakthrough of WS column was very steeper and breakthrough time was smaller. According to Equ. (1) and (2), values of qe about WS and NR-loaded WS were calculated and they were 6.25 and $49.7 \mathrm{mg} / \mathrm{g}$, respectively. So the adsorption capacity of WS for CR removal was significantly improved after NR adsorption. This implied that no additional reagent for regeneration As there is negative charge at surface of WS and action between WS and anionic ions is repulsive, the adsorption quantity of WS for anionic pollutants is often lower.

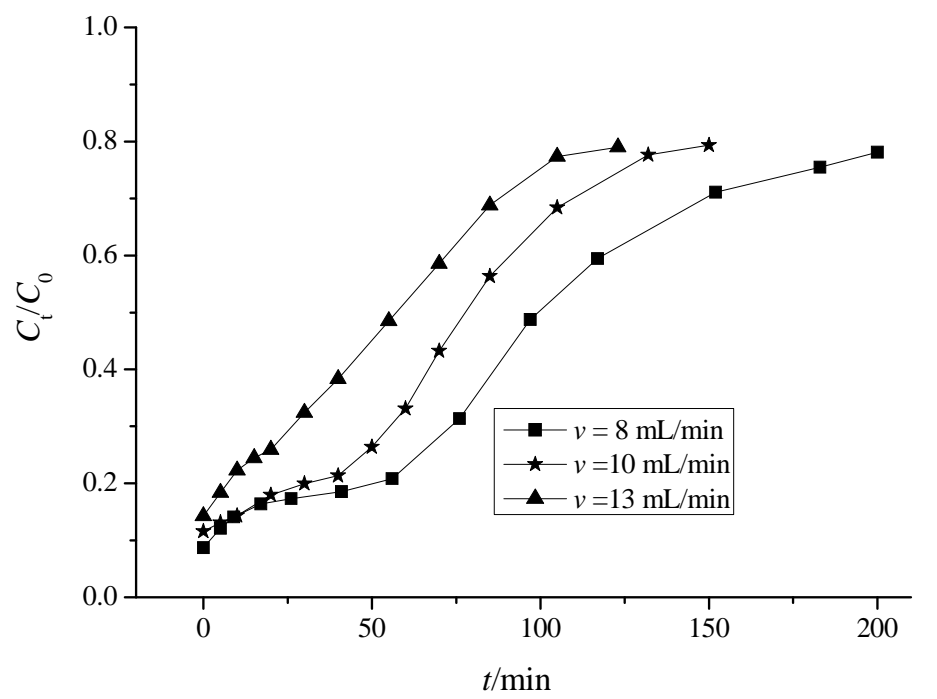

Fig. 2. Effect of flow rate on breakthrough curves $\left(C_{0}=50 \mathrm{mg} / \mathrm{L}, \mathrm{Z}=8 \mathrm{~cm}\right)$.

After binding NR, the surface structures of WS have been changed after covered by a layer of NR, so the repulsive force is screened. So NR-loaded WS can effectively adsorb CR from solution and the potential of the disused sorbent is further exploited.

Effect of flow rate on breakthrough curves. The breakthrough curves at various flow rates were shown in Fig. 2. It was shown that breakthrough generally occurred faster with higher flow rate. Breakthrough time reaching saturation was increased significantly with a decreased in the flow rate. When at a low rate of influent, CR had more time to contact with NR-loaded WS and it resulted in 
higher removal of NR ions from solution in column.

Effect of bed height on breakthrough curves. The breakthrough curves at different bed depth were shown in Fig. 3. From Fig. 3, as the bed height increases, CR had more time to contact with NT-loaded WS that resulted in higher removal efficiency of CR ions in column. So the higher bed column results in a decrease in the solute concentration in the effluent at the same time. The slope of breakthrough curve decreased with increasing bed height, which resulted in a broadened mass transfer zone [12].

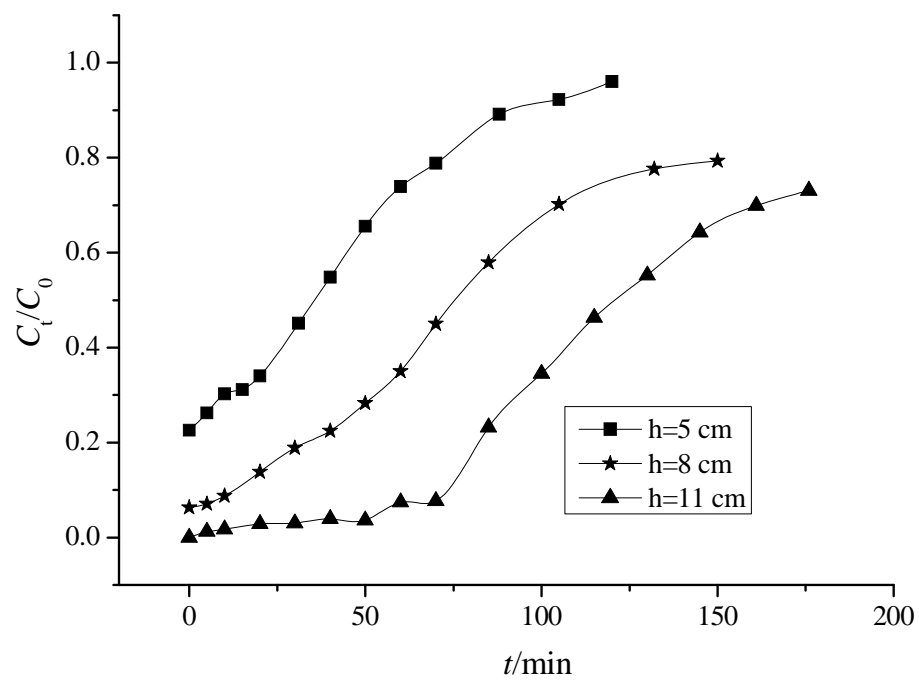

Fig. 3. Effect of bed depth on breakthrough curves $\left(C_{0}=50 \mathrm{mg} / \mathrm{L}, v=10 \mathrm{~mL} / \mathrm{min}\right)$.

Application of Thomas models. Table 1 listed the parameters of Thomas model and determined coefficients by nonlinear regression analysis using least square method. The values of $q_{\mathrm{e}}$ at various conditions were also listed in table 1.

It was found that Thomas model described the breakthrough curves properly for higher correlation coefficient. In addition, the simulated CR uptakes $\left(q_{0}\right)$ were also very close to the values $\left(q_{\mathrm{e}}\right)$ calculated from Eq. (2). These implied that Thomas models can be used to fit the experimental data and to predict the column adsorption quantity. From Table 1 and Fig. 1-3, higher flow rate and lower bed depth were disadvantage of adsorption quantity $\left(q_{0}\right)$ for CR on NR-loaded WS column.

\section{Conclusions}

On the base of the results, the following conclusion can be drawn:

a. NR-loaded WS can effectively recycled to remove CR from solution without further treatment.

b. The influent CR concentration, flow rate of solution and bed depth significantly affect the breakthrough curve.

c. Thomas model was available to fit the process and predict the column adsorption quantity.

Table 1 Parameters of Thomas model by nonlinear regressive analysis

\begin{tabular}{ccccccc}
\hline $\begin{array}{c}C_{0} / \\
(\mathrm{mg} / \mathrm{L})\end{array}$ & $\begin{array}{c}v / \\
(\mathrm{mL} / \mathrm{min})\end{array}$ & $\begin{array}{c}\mathrm{Z} / \\
(\mathrm{cm})\end{array}$ & $\begin{array}{c}k_{\mathrm{Th}} / \\
(\mathrm{mL} / \mathrm{mg} \min )\end{array}$ & $\begin{array}{c}q_{0} / \\
(\mathrm{mg} / \mathrm{g})\end{array}$ & $\begin{array}{c}q_{\mathrm{e}} / \\
(\mathrm{mg} / \mathrm{g})\end{array}$ & $R^{2}$ \\
\hline 50.0 & 8.0 & 8.0 & 0.353 & 55.3 & 56.5 & 0.978 \\
50.0 & 10.0 & 8.0 & 0.513 & 51.2 & 50.1 & 0.982 \\
50.0 & 13.0 & 8.0 & 0.522 & 47.7 & 48.2 & 0.988 \\
30.0 & 10.0 & 8.0 & 0.694 & 45.9 & 45.7 & 0.988 \\
80.0 & 10.0 & 8.0 & 0.432 & 51.8 & 56.7 & 0.964 \\
50.0 & 10.0 & 5.0 & 0.742 & 34.1 & 39.9 & 0.995
\end{tabular}




$\begin{array}{lllllll}50.0 & 10.0 & 11.0 & 0.617 & 62.8 & 60.3 & 0.971\end{array}$

\section{Conclusions}

On the base of the results, the following conclusion can be drawn:

a. NR-loaded WS can be effectively recycled to remove CR from solution.

b. The influent CR concentration, flow rate of solution and bed depth significantly affect the breakthrough curve.

c. Thomas model was available to fit the experimental data and predict the column adsorption quantity.

\section{Acknowledgements}

This work was supported by the National Natural Science Foundation of China for undergraduate cultivation in basic science (J1210060).

\section{References}

[1] A. Bhatnagar and M. Sillanpaa: Chem. Eng. J. Vol. 157 (2010), p. 277.

[2] G. Crini: Bioresour. Technol. Vol. 97 (2006), p. 1061.

[3] J.Y. Song, W.H. Zou, Y.Y. Bian, F.Y. Su and R.P. Han: Desalination Vol. 265 (2011), p. 119.

[4] Y.J. Wu, L.J. Zhang, C.L. Gao, J.Y. Ma, X.H. Ma and R.P. Han: J. Chem. Eng. Data Vol.54 (2009), p. 322.

[5] R.P. Han, D.D. Ding, Y.F. Xu, W.H. Zou, Y.F. Wang, Y.F. Li and L.N. Zou: Bioresour. Technol. Vol. 99 (2008), p. 2938.

[6] R.P. Han, Y.F. Wang, P. Han, J. Shi, J. Yang and Y.S. Lu: J. Hazard. Mater. Vol. 137 (2006), p. 550.

[7] R.P. Han, W.H. Zou, W.H. Yu, S.J. Cheng, Y.F. Wang and Jie Shi: J. Hazard. Mater. Vol. 141 (2007), p. 156.

[8] T. Zhou, W.Z. Lu, L.F. Liu, H.M. Zhu, Y.B. Jiao, S.S. Zhang and R.P. Han: J. Mol. Liq. Vol. 211 (2015), p. 909.

[9] X. Xu, B.Y. Gao, B. Jin and Qi.Y. Yue: J. Mol. Liq. Vol. 215 (2016), p. 565.

[10] R.P. Han, L.J. Zhang, C. Song, M.M. Zhang, H.M. Zhu and L.J. Zhang: Carbohyd. Polym. Vol. 79 (2010) p. 1140.

[11] W.X. Zhang, H.J. Li, X.W. Kan, L. Dong, H. Yan, Z.W. Jiang, H. Yang, A.M. Li,R.S. Cheng: Bioresour. Technol. Vol. 117 (2012), p. 40.

[12] Z. Akzu and F. Gonen: Process Biochem. Vol. 39 (2004) p.599.

[13] R.P. Han, L.N. Zou, X. Zhao, Y.F. Xu, F. Xu, Y.L. Li and Y. Wang: Chem. Eng. J. Vol. 149 (2009), p. 123. 